\title{
Nanophase-segregation in the dielectric
}

\author{
layer enhances the charge storage
}

capacity of polymeric electrochemical

\section{supercapacitors}

Nuria Borras, ${ }^{1}$ Francesc Estrany, ${ }^{1,2, *}$ and Carlos Alemán ${ }^{1,2, *}$

${ }^{1}$ Departament d'Enginyeria Química, EEBE, Universitat Politècnica de Catalunya, C/ Eduard Maristany, 10-14, Ed. I2, 08019, Barcelona, Spain.

${ }^{2}$ Barcelona Research Center in Multiscale Science and Engineering, Universitat Politècnica de Catalunya, C/ Eduard Maristany, 10-14, 08019, Barcelona, Spain 


\begin{abstract}
Properties related with the charge storage capacity have been evaluated for threelayered films made with two sheets of poly(3,4-ethylenedioxythiophene) separated by an sheet of poly(N-methylpyrrole) or poly(3,4-ethylenedioxythiophene-co-Nmethylpyrrole) (31-PEDOT/PNMPy or 31-PEDOT/P(EDOT-co-NMPy), respectively). The most distinctive trend of the copolymer, which shows electrochemical properties intermediate between those of the two homopolymers, is the formation of a biphasic structure, EDOT- and NMPy-rich blocks organizing separately. The ability to exchange charge reversibly is higher for 31-PEDOT/P(EDOT-co-NMPy) than for 31PEDOT/PNMPy, the electroactivity and electrostability of such two 3-layered films being significantly better than that of single-layered PEDOT. Advantages of 31PEDOT/P(EDOT-co-NMPy) are mainly based on the nanophase-segregated structure of the copolymer. Thus, the intermediate layer can be considered as random disposition of ultrathin dielectrics having nanometric length and width. In terms of charge storage, the intermediate layer of 31-PEDOT/P(EDOT-co-NMPy) can be viewed as a thin reservoir filled of heterogeneously distributed nanometric supercapacitors that are connected in series among them and in parallel to the PEDOT layers. The superiority of 31PEDOT/P(EDOT-co-NMPy) as organic electrochemical supercapacitor compared to other 3-layered systems, has been proved by powering a red LED bulb.
\end{abstract}

Keywords: Conducting polymer; Copolymer; Multilayer; Plane capacitor; Poly(3,4ethylenedioxythiophene); Polypyrrole 


\section{INTRODUCTION}

With the rapid depletion of fossil fuels and increasing environmental pollution, there is an urgent need not only for sustainable and clean sources of energy but for efficient technologies associated with energy conversion and storage. Regarding to the latter, electrochemical supercapacitors (ESCs), which were designed to bridge the gap between batteries and capacitors, are attracting significant attention due to their high power density and long life-cycle [1-6].

According to the mechanism of energy storage, ESCs are generally divided into two types: electrochemical double-layer capacitors, in which capacitance arises from charge accumulation in the electrode/electrolyte interface, and pseudocapacitors, which are based on the fast and reversible redox reaction at the surface of electroactive materials [7-9]. In general, the capacitance of pseudocapacitors is much higher than electrochemical double-layer capacitors. The electrode materials for electrochemical double-layer capacitors mainly include carbonaceous species [10,11], while transition metal oxides $[10,12]$ and conducting polymers $[10,13,14](\mathrm{CPs})$ are commonly used as electrode materials for pseudocapacitors. Because of its excellent performance, poly(3,4-ethylenedioxythiophene (PEDOT; Scheme 1) films are frequently used as electrodes for the fabrication of organic electrochemical supercapacitors (OESCs) [1521]. PEDOT, which is commercialized under the trade name of Clevios ${ }^{\mathrm{TM}}$ (Heraeus) and Baytron $^{\circledR}$ (from H. C. Starck), exhibits low band gap, easiness to stabilize the oxidized (also named p-doped) state, high conductivity and electrochemical activity, and excellent pseudocapacitance behavior [15-27]. The latter been attributed to the coexistence of both $(i)$ the redox mechanism, according to which charge is stored by electron transfer from the dopant to the polymer (i.e. ion pairing stabilizes the charge); 
and (ii) the double-layer behavior of doped PEDOT chains, which allows resonance throughout the conjugated structure of the polymeric backbone [15-22].

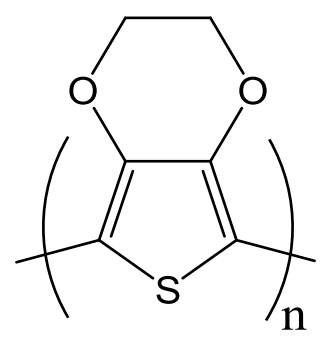

Scheme 1. Chemical structure of PEDOT

In an effort to further improve mechanical, thermal, optical and/or electrical properties of PEDOT-based electrodes, this $\mathrm{CP}$ has been combined with other compounds, as for example polymers (e.g. polyimines) [28,29], inorganic salts (e.g. $\mathrm{MnO}_{2}$ ) [30,31], carbonaceous compounds (e.g. graphene) [32,33], and clays (e.g. montmorillonite) [34,35], to form homogeneous composites. An alternative approach to create synergies is the preparation of multi-layered heterogeneous composites combining alternated sheets of PEDOT and another material, as for example carbon nanotubes [36], graphene [37], polyelectrolytes [38], metallic nanowires [39], metallic oxides [40], and even other CPs [17,41].

Regarding with the latter, a few years ago we used the layer-by-layer (LbL) electrodeposition technique to prepare 3-layered conducting systems based on PEDOT and poly(N-methylpyrrole) (PNMPy) layers arranged alternatively [17,42], hereafter abbreviated 31-PEDOT/PNMPy. These 3-layered materials showed better electrochemical properties and higher capacitance than each of the two individual CPs. This phenomenon was attributed to a synergistic effect produced by the favorable interaction between the PEDOT and PNMPy layers at the corresponding interfaces, the PNMPy intermediate sheet promoting the dielectric breakage effect (i.e. the electrical 
conductivity is $\sim 10^{5} \mathrm{~S} / \mathrm{cm}$ higher for PEDOT than for PNMPy). Indeed, the coupling between PEDOT and PNMPy was found to be, in terms of enhancement of the electrochemical properties, significantly more positive in 3-layered films than the interaction between the two monomers in the corresponding copolymers, poly(EDOTco-NMPy) [43]. In addition, the specific capacitance of 31-PEDOT/PNMPy was found to be significantly higher than that of pure PEDOT ( $\mathrm{SC}=90$ and $41 \mathrm{~F} / \mathrm{g}$, respectively). More recently, 31-PEDOT/PNMPy systems were improved by incorporating exfoliated montmorillonite (MMT) at the intermediate PNMPy layer (i.e. 31PEDOT/P(NMPy+MMT) [44]. This inorganic additive, which was incorporated using an in situ polymerization procedure, enhanced the sensitivity to the dielectric breakage, activating the electrochemical and capacitive properties of the composite.

In this work we examine a different approach, which is based on the substitution of PNMPy by poly(EDOT-co-NMPy) as intermediate sheet of PEDOT-based 3-layered systems. The advantage of this new configuration, denoted 31-PEDOT/P(EDOT-coNMPy), is not only the elimination of the inorganic additive with respect to 31PEDOT/P(NMPy+MMT) but also the benefits associated to the incorporation of relevant nanofeatures, which result in a significant improvement of the properties with respect to 31-PEDOT/PNMPy. These nanofeatures are based on the biphasic organization poly(EDOT-co-NMPy) chains, transforming the intermediate dielectric layer into a random disposition of many dielectric nanolayers. The higher performance of 31-PEDOT/P(EDOT-co-NMPy) has been demonstrated by comparing its ability to store charge with that of 31-PEDOT/PNMPy and also by constructing OESCs with 31PEDOT/P(EDOT-co-NMPy), 31-PEDOT/PNMPy and 31-PEDOT/P(NMPy+MMT). The advantageous outcome of the 31-PEDOT/P(EDOT-co-NMPy)-OESC as energy storage device is evidenced with a practical application using LED bulb. 


\section{METHODS}

Materials. 3,4-ethylenedioxythiophene (EDOT), N-methylpyrrole (NMPy) and acetonitrile were purchased from Sigma-Aldrich (Spain) and used as received. Anhydrous lithium perchlorate $\left(\mathrm{LiClO}_{4}\right)$ was purchased from Sigma-Aldrich (Spain) and was stored in an oven at $80{ }^{\circ} \mathrm{C}$ before use in the electrochemical trials. All chemicals were analytical reagent grade.

Synthesis of PEDOT, PNMPy and poly(EDOT-co-NMPy) layers. Layers were prepared by chronoamperometry (CA) under a constant potential of $1.40 \mathrm{~V}$ using a three-electrode one-compartment cell under nitrogen atmosphere (99.995\% in purity) at $25{ }^{\circ} \mathrm{C}$. Steel AISI 316 sheets of $2 \times 2 \mathrm{~cm}^{2}$ area were employed as working and counter electrodes. To avoid interferences during the electrochemical analyses, before each trial the working and counter electrodes were cleaned with ethanol, after that with acetone, and dried in an air-flow. The reference electrode was an $\mathrm{Ag} \mid \mathrm{AgCl}$ electrode containing a $\mathrm{KCl}$ saturated aqueous solution $\left(\mathrm{E}^{\mathrm{o}}=0.222 \mathrm{~V}\right.$ vs. standard hydrogen electrode at $\left.25^{\circ} \mathrm{C}\right)$. All the potentials reported in this work are referred to the $\mathrm{Ag} \mid \mathrm{AgCl}$ electrode. The cell was filled with $40 \mathrm{~mL}$ of $10 \mathrm{mM}$ monomer solution in acetonitrile containing $0.1 \mathrm{M}$ $\mathrm{LiClO}_{4}$ as doping electrolyte. The total concentration of monomers in the solution used to prepare the copolymer was kept fixed at $10 \mathrm{mM}$, even though the EDOT:NMPy molar ratio was 2:1. In order to evaluate the influence of the thickness on the electrochemical properties, layers were generated using polymerization times $(\theta)$ ranging from 100 to $450 \mathrm{~s}$. All electrochemical experiments were conducted on a PGSTAT302N AUTOLAB potentiostat-galvanostat connected to a PC computer and controlled through the NOVA 1.6 software. 
Electrochemical characterization. In all cases the electrochemical properties were studied using an acetonitrile solution with $0.1 \mathrm{M} \mathrm{LiClO}_{4}$. The electroactivity and electrostability were determined by cyclic voltammetry (CV). The initial and final potentials were $-0.5 \mathrm{~V}$, and the reversal potential was $1.6 \mathrm{~V}$. A scan rate of $50 \mathrm{mV} \cdot \mathrm{s}^{-1}$ was used. The ability to exchange charge reversibly (i.e. electroactivity) and the electrochemical stability (i.e. electrostability) were determined through direct measure of the anodic and cathodic areas in the control voltammograms using NOVA software.

The loss of electroactivity (LEA, in \%) was expressed as:

$$
\mathrm{LEA}=\frac{\Delta \mathrm{Q}}{\mathrm{Q}_{\mathrm{II}}} \cdot 100
$$

where $\Delta \mathrm{Q}$ is the difference of voltammetric charges (in $\mathrm{C}$ ) between the second and the last cycle, and $\mathrm{Q}_{\mathrm{II}}$ is the voltammetric charge corresponding to the second cycle.

Charge-discharge assays were performed using a two-electrode cell. The latter was connected to an ammeter (in series) and a voltmeter (in parallel) to measure the intensity and voltage, respectively (Scheme 2). A HAMEG HM7042-4 power supply of 6V was used for the charge process. In order to regulate the intensity during both the charge and discharge processes, resistances of $10 \mathrm{k} \Omega$ and $1 \mathrm{k} \Omega$, respectively, were introduced into the corresponding electric circuit (Scheme 2).

Microscopy. Atomic force microscopy (AFM) images were obtained with a Molecular Imaging PicoSPM using a NanoScope IV controller under ambient conditions. The tapping mode AFM was operated at constant deflection. The row scanning frequency was set to $1 \mathrm{~Hz}$. AFM measurements were performed on various parts of the films, which provided reproducible images similar to those displayed in this work. The scan window sizes used in this work were $5 \times 5 \mu \mathrm{m}^{2}$. The statistical application of the NanoScope Analysis software was used to determine the root mean 
square roughness (RMS Rq), which is the average height deviation taken from the mean data plane.

Scanning electron microscopy (SEM) studies were performed to examine the surface morphology of PEDOT, PNMPy and poly(EDOT-co-NMPy) films. Dried samples were placed in a Focussed Ion Beam Zeis Neon 40 scanning electron microscope operating at $3 \mathrm{kV}$, equipped with an EDX spectroscopy system.

(a)

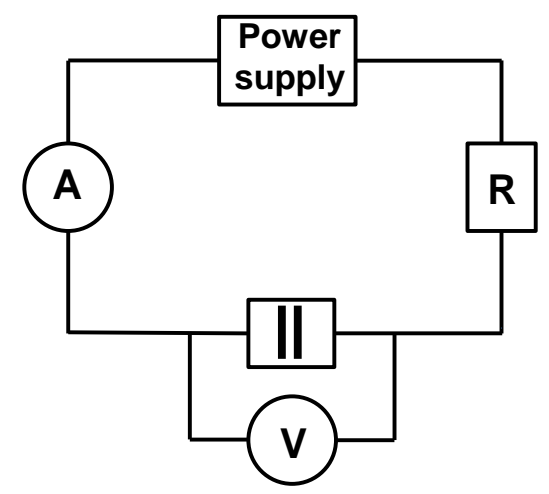

(b)

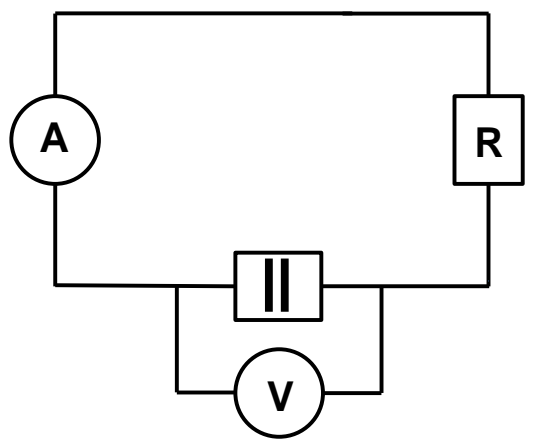

Scheme 2. Electric circuit used in (a) charge and (b) discharge assays.

\section{RESULTS AND DISCUSSION}

\section{Preparation and characterization of the individual layers}

PEDOT, PNMPy and poly(EDOT-co-NMPy) layers were prepared by chronoamperometry (CA) applying a constant potential of $1.40 \mathrm{~V}$ during the polymerization time $\theta$. The latter, which is specified in each case, has been used to 
regulate the thickness of the layer (Table 1). During the polymerization process the concentration of monomer was kept fixed at $10 \mathrm{mM}$ in all cases, the EDOT:NMPy molar ratio used for the preparation of the copolymer being 2:1.

Cyclic voltammograms recorded for PEDOT ( $\theta=400 \mathrm{~s})$, PNMPy $(\theta=100 \mathrm{~s})$ and poly(EDOT-co-NMPy) ( $\theta=100 \mathrm{~s})$ films after two and fifty consecutive oxidationreductions cycles are compared in Figure 1a. Inspection of the $2^{\text {nd }}$ voltammogram evidences that the electrochemical activity decreases as follows: PEDOT > poly(EDOT-co-NMPy) >> PNMPy. The remarkable difference between PEDOT and PNMPy reduction is due to a combination of factors. Firstly, PEDOT chains are exclusively formed by $\alpha, \alpha$-linkages since the dioxane ring fused onto the thiophene ring occupies the $\beta$-positions of the latter, whereas PNMPy chains grow forming branched structures due to the inter-ring coupling in the available $\beta$ - and $\beta$ '-positions of the NMPy units. In addition, PEDOT chains are completely planar due to both the restriction imposed by the fused dioxane ring and to the electron-donating effects provided by the oxygen atoms contained in such cyclic substituents (i.e. selfrigidification effect), while inter-ring dihedral angles deviate by around $30^{\circ}$ in PNMPy chains [45]. These two phenomena promote the gain in aromaticity and electrochemical activity in PEDOT films compared to PNMPy. Poly(EDOT-co-NMPy) presents an intermediate situation, which explains the behavior of the copolymer with respect to the two homopolymers. Secondly, the thickness and, therefore, the polymer mass is significantly higher for PEDOT than for PNMPy (Table 1). This must be attributed to the higher polymerization time used to prepare the former (i.e. $400 \mathrm{~s} v s .100 \mathrm{~s}$ ) since the mobility of the two monomers is very similar (i.e. the diffusion coefficient is $5.43 \times 10^{-5}$ $6.84 \times 10^{-5} \mathrm{~cm}^{2} / \mathrm{s}$ for EDOT and NMPy, respectively) [43]. 
After 50 consecutive redox cycles, the electrochemical activity of PEDOT remains very high, whereas that of poly(EDOT-co-NMPy) experiences a drastic reduction (Figure 1a). Analysis of the variation of the accumulated voltammetric charge $(Q)$ with the number of redox cycles supports this observation (Figure 1b). Although $Q$ decreases with increasing number of cycles in all cases, this reduction strongly depends on the material. Thus, the copolymer experiences a continuous and sharp reduction during the whole process, the loss of electroactivity (LEA) after 50 cycles being of $73 \%$. The reduction is more progressive for PNMPy, which exhibits a final LEA value of $47 \%$. Finally, PEDOT, which displays the highest $Q$, preserves $84 \%$ of the charge after 50 cycles $(\mathrm{LEA}=16 \%)$.

The topography and morphology of the different polymers were studied by AFM and SEM, respectively. Representative height AFM images and high resolution SEM micrographs of PEDOT, PNMPy and poly(EDOT-co-NMPy) films prepared using $\theta=$ $100 \mathrm{~s}$ are compared in Figure 2. PEDOT films display a heterogeneous surface with multiple agglomerates, interconnected through thin fiber-like structures, defining tortuous pores. These features determine root mean square roughness (RMS Rq) of PEDOT that is the relatively high, ranging from 268 to $300 \mathrm{~nm}$. Obviously, the RMS Rq increases with the polymerization time (not shown). In contrast, PNMPy presents a compact, homogeneous and globular morphology without pores or fiber-like structures separating the relatively small corpuscles observed at the surface. Consistently, the RMS Rq, which is comprised between 65 and $75 \mathrm{~nm}$, shows a significant reduction with respect to PEDOT films generated using the same $\theta$ value.

Inspection to the surface topography and morphology obtained for poly(EDOT-coNMPy) (Figure 2c) reveals a situation that is intermediate between those exhibited by the two homopolymers. Thus, the morphology of the copolymer films, which exhibit 
RMS Rq values comprised between 80 and $90 \mathrm{~nm}$, can be described as the superposition of the homogeneous compact structure identified for PNMPy and a clustered heterogeneous distribution found for PEDOT. This feature suggests that the EDOT- and NMPy-blocks in the copolymer distribute in separated phases, capturing the most characteristic structural trends of the two homopolymers. This hypothesis is supported by the contrast in the phase image included in Figure $2 \mathrm{c}$, which allows to distinguish between two phases in poly(EDOT-co-NMPy) films. The bright areas are associated with the EDOT-rich phase (i.e. PEDOT with some dissolved PNMPy), whereas the dark areas represent the NMPy-rich phase (i.e. PNMPy with some dissolved PEDOT). It is worth noting that the phase segregation displayed in Figure $2 \mathrm{c}$ is consistent with early quantum mechanical calculations, which predicted a block microstructure for poly(EDOT-co-NMPy) [46]. The segregation of the copolymer in NMPy- and EDOTrich phases is expected to have major implications in the capacitance performance of 31$\mathrm{PEDOT} / \mathrm{P}(\mathrm{EDOT}-\mathrm{co}-\mathrm{NMPy}$ ) (see below). Indeed, the observed phase segregation is fully consistent with the specific capacitance of the copolymer film ( $S C=72 \mathrm{~F} / \mathrm{g}$ ), which is higher than those of films made with PEDOT or PNMPy homopolymers $(S C=60$ or $26 \mathrm{~F} / \mathrm{g})$.

\section{Characterization of 3-layered systems}

31-PEDOT/PNMPy and 31-PEDOT/P(EDOT-co-NMPy) films were prepared using $\theta_{1,3}=200 \mathrm{~s}$ and $\theta_{2}=100 \mathrm{~s}$, where $\theta_{1,3}$ refers to the polymerization time of the internal and external PEDOT layers and $\theta_{2}$ is the polymerization time of the intermediate PNMPy or poly(EDOT-co-NMPy) layer. The thickness of the resulting films is included in Table 1, while Figure 3a compares the cyclic voltammograms and the variation of the voltammetric charge against the number of redox cycles obtained for the 
3-layered films with those achieved using individual PEDOT films ( $\theta=400 \mathrm{~s})$. As it can be seen, the electrochemical activity of the 3-layered systems is higher than that of individual PEDOT films, this feature being especially remarkable for 31PEDOT/P(EDOT-co-NMPy). Similarly, the electrochemical stability of the 3-layered films is much higher than that of PEDOT. The LEA after 50 cycles is $1 \%$ and $2 \%$ for $31-$ PEDOT/PNMPy and 31-PEDOT/P(EDOT-co-NMPy), respectively, this value increasing to $16 \%$ for PEDOT.

Detailed comparison between the voltammetric charges of 31-PEDOT/PNMPy and 31-PEDOT/P(EDOT-co-NMPy) reflects that the electroactivity is $12 \%$ lower for the former than for the latter (Figure 3b). After 50 redox cycles, differences between the voltammetric charges of 31-PEDOT/P(EDOT-co-NMPy) and 31-PEDOT/PNMPy increases to $15 \%$. Figure $3 \mathrm{c}$ compares the chronopotentiometric response (i.e. potential vs time) of the 3-layered systems with that of that of PEDOT at a constant anodic current of $1 \mathrm{~mA} / \mathrm{cm}^{2}$. PEDOT reaches a voltage of $1.50 \mathrm{~V}$ after $500 \mathrm{~s}$, while 31PEDOT/PNMPy and 31-PEDOT/P(EDOT-co-NMPy) arrived at 1.44 and $1.39 \mathrm{~V}$, respectively. Accordingly, the presence of PNMPy and poly(EDOT-co-NMPy) intermediate layers causes a reduction of 0.06 and $0.11 \mathrm{~V}$, respectively, evidencing that the oxidation of the film is lower for the 3-layered systems than for PEDOT). This behavior is fully consistent with the ability to exchange charge reversibly of 3-layered films displayed in Figures 3a-b. On the other hand, the specific capacitances of 31PEDOT/PNMPy and 31-PEDOT/P(EDOT-co-NMPy) are $S C=57$ and $70 \mathrm{~F} / \mathrm{g}$, respectively.

Differences between 31-PEDOT/PNMPy and 31-PEDOT/P(EDOT-co-NMPy) must be exclusively attributed to the structure of the copolymer, in which NMPy-rich blocks organize in nanophases separated from PEDOT-rich blocks (Figure 2a). As is 
schematically displayed in Figure 4a, the intermediate layer in 31-PEDOT/PNMPy behaves as a homogeneous and continuous dielectric with PNMPy chains organized in a single phase of micrometric thickness (i.e. the thickness of the PNMPy layer, $1.5 \mu \mathrm{m}$, corresponds to the separation between the two conducting PEDOT electrodes). Instead, in 31-PEDOT/P(EDOT-co-NMPy), the central copolymer layer behaves heterogeneously, as is illustrated in Figure 4b. More specifically, the small PNMPy-rich phases, which are organized randomly, act as nano-dielectric layers. Besides, electrodes include not only the two micrometric PEDOT layers but also the small PEDOT-rich phases within the copolymer layer. According to these nanofeatures, the poly(EDOTco-NMPy) layer is not only the dielectric of a micrometric PEDOT supercapacitor but also is a thin reservoir filled of heterogeneously distributed nanometric supercapacitors that are connected in series among them and in parallel to the former one. Moreover, the nanometric dimension of PNMPy-rich phases (Figure 4b) enhances the charge storage capacity of the external PEDOT layers, since it increases with decreasing separation between them.

\section{Optimization of the 3-layered configuration}

A systematic study varying the thickness of the intermediate PNMPy layer was carried to prove that 31-PEDOT/PNMPy behave plane-capacitor made of two conducting PEDOT layers separated by a small distance (dielectric intermediate layer). For this purpose, 31-PEDOT/PNMPy films, which were obtained using $\theta_{2}=100,300$ and $450 \mathrm{~s}$ while $\theta_{1,3}$ was kept fixed $100 \mathrm{~s}$, were connected to electric circuits like those displayed Figure S1 for charging-discharging actions. Figure 5a displays the temporal evolution of the accumulated voltage across the films for representative charging processes. The voltage drops with increasing thickness of the PNMPy layer, reflecting a 
shielding effect that increases with the distance between the PEDOT layers. This phenomenon was corroborated by measuring both the temporal evolution of the current (I- $t$ curve) and the stored charge (Figures 5b-c). Both the current flow, which is proportional to the rate of change of voltage across the capacitor, and the stored charge, which was determined during discharging actions, decrease with the increasing thickness of the PNMPy layer, even though no linear proportionality was appreciated. These results demonstrate that the smaller distance between the PEDOT layers is, the lower polarization of PEDOT layers and the larger capacitance are obtained. It should be mentioned that studies with 31-PEDOT/P(NMPy-co-EDOT) films using $\theta_{2}>100 \mathrm{~s}$ were not possible due to the poor stability of the copolymer layer. This drawback was attributed to the limitations associated with the phase segregation process when the thickness of the film exceeds a threshold value $(\sim 1.5 \mu \mathrm{m})$.

On the other hand, Figures 5d-f display the results obtained for 31-PEDOT/PNMPy films prepared using $\theta_{1,3}=100,300$ and $450 \mathrm{~s}$, while $\theta_{2}=100 \mathrm{~s}$ in all cases to keep the distance between the two conducting layers fixed. As it was expected, the charge storage capacity improves with the increasing thickness of the conducting layers. The charge released during the discharging process for the system with $\theta_{1,3}=450 \mathrm{~s}$ is $46 \%$ and $26 \%$ higher than those of systems with $\theta_{1,3}=100$ and $300 \mathrm{~s}$, respectively, while the potential is lower for the former.

Considering that in the highest capacity to store charge was obtained for films with the thickest PEDOT layers and the thinnest PNMPy layer, Figure 6 compares the behavior of 31-PEDOT/PNMPy and 31-PEDOT/P(EDOT-co-NMPy) prepared using $\theta_{1,3}=450 \mathrm{~s}$ and $\theta_{2}=100 \mathrm{~s}$. The latter system reaches the highest potential during the whole charging process. For example, after 6 min the charging potential of 31PEDOT/poly(EDOT-co-NMPy) and 31-PEDOT/PNMPy is 1.7 and $1.1 \mathrm{~V}$, respectively, 
the voltages reached after 17 min being 2.0 and $1.6 \mathrm{~V}$. Furthermore, the charge released during the discharging process is $20 \%$ higher for 31-PEDOT/poly(EDOT-co-NMPy) evidencing that the copolymer improves the capacitor performance. On the other hand, charge-discharge curves, which are displayed in Figure 6d, have been to evaluate the Coulombic efficiency $(\eta, \%)$ as the ratio between the discharging and charging times. According to the rest observations, the Coulombic efficiency of 31PEDOT/poly(EDOT-co-NMPy) $(\eta=70 \%)$ was slightly higher than that of 31$\operatorname{PEDOT} / \mathrm{PNMPy}(\eta=65 \%)$.

The better behavior of 31-PEDOT/P(EDOT-co-NMPy) have been attributed to the microstructure of the poly(EDOT-co-NMPy) layer [43], which segregates into NMPyand EDOT-rich phases. This biphasic organization induces the apparition of multiple nanometric NMPy-rich phases, which act reducing the distance between conducting PEDOT layers. Within this context, 31-PEDOT/P(EDOT-co-NMPy) films could be interpreted as 31-PEDOT/PNMPy films in which the intermediate layer consists of a heterogeneous distribution of ultra-thin layers having nanometric-scale also in length.

\section{Practical application: Proof of concept with a LED bulb}

In order to show the potential application of 31-PEDOT/P(EDOT-co-NMPy) as OESCs devices, four electrodes consisting on 3-layered polymeric films deposited onto steel AISI 316 sheets were distributed in two cells filled with a $0.1 \mathrm{M} \mathrm{LiClO}_{4}$ acetonitrile solution, which were used to power a red LED bulb. Considering that the working potential of the LED bulb is $1.65-1.70 \mathrm{~V}$, the operational potential of a single cell $(0.35-0.30 \mathrm{~V})$ was considered too low to illustrate the effect of the OESC in powering the bulb. Accordingly, the two cells were connected in series, as is displayed 
in Figure $7 \mathrm{a}$, supplying an initial potential of up to $4.0 \mathrm{~V}$. The electrodes acted as the anode and the cathode of the OESC.

The circuit consisted in a power supply of $12.6 \mathrm{~V}$ for charging, an ammeter connected in series to register variation of intensity during the discharge, and a voltmeter connected in parallel to register the variation of potential. When connecting $n$ identical electrodes in series, each voltage threshold $V$ is combined to theoretically result in a voltage threshold equal to $n \cdot V$. Nevertheless, in practice, combining electrodes in series also leads to an equivalent series resistance and, slightly different leakage current may induce voltage across each one to differ, thus leading to a resulting voltage threshold lower than the theoretical value.

Figure $7 \mathrm{~b}$ displays the variation of the voltage during the charging-discharging processes of the OESC, the complete discharge of the device being achieved using a resistance of $1 \mathrm{k} \Omega$. As it can be seen, a $260 \mathrm{~s}$ charging process resulted in voltage window of $4 \mathrm{~V}$, while only $32 \mathrm{~s}$ were required to reach an open circuit voltage lower than $1.5 \mathrm{~V}$ during the discharging. The charge accumulated and released during this processes were 181.2 and $170.8 \mathrm{mC}$, respectively (Figure 7c), evidencing that the latter corresponds to $94 \%$ of the former. On the other hand, the LED was lit for more than 155 s when using OESC 31-PEDOT/P(EDOT-co-NMPy) (Figure 7d). This interval of time was higher than that achieved using the devices constructed with 31-PEDOT/PNMPy $(<$ $135 \mathrm{~s})$ and 31-PEDOT/clay ( $<60 \mathrm{~s})$ electrodes. Accordingly, the nanofeatures observed in dielectric layers constructed using poly(EDOT-co-NMPy) provide a significant practical improvement with respect to those obtained using micrometric PNMPy films or inorganic clays (i.e. montmorillonite), which were introduced in PEDOT layers using a previously reported strategy for the incorporation of exfoliated clays by in situ anodic polymerization [34]. The very low performance of OESCs based on 31-PEDOT/clay 
electrodes has been attributed to the completely inert nature of the clay. Furthermore, it should be mentioned that the same 31-PEDOT/P(EDOT-co-NMPy)-based device was used to repeat the recharge process by more than 10 times, which were distributed in a month. In all cases the OESC successfully powered the LED bulb without any detriment in its performance. This feature proved the stability of the OESC prepared using the copolymer as dielectric layer.

On the basis of our previous studies on multilayered systems [42], 5- and 7-layered electrodes based on alternated layers of PEDOT and poly(EDOT-co-NMPy) are expected to exhibit higher redox capacity and charge store ability than 3-layered ones. However, the margin of improvement in 5- and 7-layered electrodes over the result of the 3-layered ones is expected to be very small in comparison with the huge increment in the cost and effort required for their preparation.

\section{CONCLUSIONS}

In summary, we demonstrate the great potential of all-CP OESCs. In particular, the performance of 31-PEDOT/PNMPy systems revealed significant enhancement of charge storage capacity when the central dielectric PNMPy layer was replaced by poly(NMPyco-EDOT). The phase-segregated structure of this copolymer, which organizes in NMPy- and EDOT-rich domains, provides nanofeatures that reduce the distance between PEDOT conducting layers. Thus, the intermediate layer of 31PEDOT/P(NMPy-co-EDOT) films behaves as a wide set of nanometric supercapacitors that are connected in series among them and in parallel to the external conducting PEDOT layers. From the performance we have achieved, we believe that the incorporation of nanofeatures through the substitution of the polymer used as dielectric by the appropriated copolymer is a promising general approach that can be used to 
improve the performance of all-CPs OESCs. Importantly, our results indicate that the copolymer phase segregation in 3-layered devices plays an important role in improving the electrochemical performance.

\section{ACKNOWLEDGEMENTS}

This work was supported by MINECO-FEDER funds (MAT2015-69367-R). Support for the research of C.A. was received through the prize "ICREA Academia" for excellence in research funded by the Generalitat de Catalunya.

\section{REFERENCES}

1. J. R. Miller, P. Simon, Materials science - Electrochemical capacitors for energy management, Science 321 (2008) 651-652.

2. C. Liu, F. Li, L. P. Ma, H. M. Cheng, Advanced materials for energy storage, Adv. Mater. 22 (2010) E28-E62.

3. Q. F. Zhang, E. Uchaker, S. L. Candelaria, G. Z. Cao, Nanomaterials for energy conversion and storage, Chem. Soc. Rev. 42 (2013) 3127-3171.

4. P. J. Hall, M. Mirzaeian, S. I. Fletcher, F. B. Sillars, A. J. R. Rennie, G. O. Shitta-Bey, G. Wilson, A. Cruden, R. Carter, Energy storage in electrochemical capacitors: designing functional materials to improve performance, Energy Environ. Sci. 13 (2013) 1238-1251.

5. P. Simon, Y. Gogotsi, Capacitive energy storage in nanostructured carbonelectrolyte systems, Acc. Chem. Res. 46 (2013) 1094-1103.

6. J. Yan, Q. Wang, T. Wei, Z. J. Fan, Recent advances in design and fabrication of electrochemical supercapacitors with high energy densities, Adv. Energy Mater. 4 (2014) 1300816. 
7. P. Simon, Y. Gogotsi, Materials for electrochemical capacitors, Nat. Mater. 7 (2008) 845-854.

8. L. L. Zhang, X. S. Zhao, Carbon-based materials as supercapacitor electrodes, Chem. Soc. Rev. 38 (2009) 2520-2531.

9. S.-K. Kim, Y. K. Kim, H. Lee, S. B. Lee, H. S. Park, Superior pseudocapacitive behavior of confined lignin nanocrystals for renewable energy storage materials, ChemSusChem 7 (2014) 1094-1101.

10. B. C. Kim, J. Y. Hong, G. G. Wallace, H. S. Park. Recent progress in flexible electrochemical capacitors: electrode materials, device configuration, and functions, Adv. Energy Mater. 5 (2015) 1500959.

11. L. Liu, Z. Niu, J. Chem, Unconventional supercapacitors from nanocarbonbased electrode materials to device configurations, Chem. Soc. Rev. 45 (2016) 43404363.

12. V. D. Nithya, N. S. Arul, Progress and development of Fe3O4 electrodes for supercapacitors, J. Mater. Chem. 4 (2016) 10767-10778.

13. S. Ghosh, T. Maiyalagan, R. N. Basu, Nanostructured conducting polymers for energy applications: towards a sustainable platform, Nanoscale 8 (2016) 6921-6947.

14. S.-K- Kim, J. Cho, J. S. Moore, H. S. Park, P. V. Braun, High-performance mesostructured organic hybrid pseudocapacitor electrodes, Adv. Funct. Mater. 26 (2016), 903-910.

15. D. Aradilla, F. Estrany, F. Casellas, J. I. Iribarren, C. Alemán, All polythiophene rechargeable batteries, Org. Electr. 15 (2014) 40-46.

16. M. M. Pérez-Madrigal, F. Estrany, E. Armelin, D. D. Díaz, C. Alemán, Towards sustainable solid-state supercapacitors: electroactive conducting polymers combined with biohydrogels, J. Mater. Chem. A 4 (2016) 1792-1805. 
17. D. Aradilla, F. Estrany, C. Alemán, Symmetric supercapacitors based on multilayers, J. Phys. Chem. C 115 (2011) 8430-8438.

18. M. M. Pérez-Madrigal, M. G. Edo, A. Díaz, J. Puiggalí, C. Alemán, Poly- $\gamma-$ glutamic acid hydrogels as electrolyte for poly(3,4-ethylenedioxythiophene)-based supercapacitors, J. Phys. Chem. C 121 (2017) 3182-3193.

19. W. Q. Zhou, J. K. Xu High-operating-voltage all-solid-state symmetrical supercapacitors based on poly(3,4-ethylenedioxythiophene)/poly(styrenesulfonate) films treated by organic solvents, Electrochim. Acta 222 (2016) 1895-1902.

20. R. Soni, B. Anothumakkool, S. Kurungot, 1D Alignment of PEDOT in a buckypaper for high-performance solid supercapacitors, ChemElectroChem 3 (2016) 1329-1336.

21. C. Karlsson, J. Nicholas, D. Evans, M. Forsyth, M. Stromme, M. Sjodin, P. C. Howlett, C. Pozo-Gonzalo, Stable deep doping of vapor-phase polymerized poly(3,4ethylenedioxythiophene)/ionic liquid supercapacitor, ChemSucChem 9 (2016) 21122121.

22. Y. Y. Lee, H. Y. Kang, S. H. Gwon G. M. Choi, S. M. Lim, J. Y. Sun, Y. C. Joo, A strain-insensitive stretchable eectronic conductor: PEDOT:PSS/acrylamide organogels, Adv. Mater. 28 (2016) 1636-1643.

23. L. B. Groenendaal, F. Jonas, D. Freitag, H. Pielartzik, J. R. Reynolds, Poly(3,4ethylenedioxythiophene) and its derivatives: Past, present, and future, Adv. Mater. 12 (2000) 481-494.

24. S. Kirchmeyer and K. Reuter, K. Scientific importance, properties and growing applications of poly(3,4-ethylenedioxythiophene), J. Mater. Chem. 15 (2005) 20772088. 
25. L. Pettersson, T. Johansson, F. Carlsson, H. Arwin, O. Inganas, Anisotropic optical properties of doped poly(3,4-ethylenedioxythiophene), Synth. Met. 101 (1999) 198-199.

26. C. Ocampo, R. Oliver, E. Armelin, C. Alemán, F. Estrany, Electrochemical synthesis of poly(3,4-ethylenedioxythiophene) on steel electrodes: Properties and characterization, J. Polym. Res. 13 (2006) 193-200.

27. C. R. G. Grenier, W. Pisula, T. J. Joncheray, K. Müllen, J. R. Reynolds, Regiosymmetric poly(dialkylphenylenedioxythiophene)s: Electron-Rich, stackable $\pi$ conjugated nanoribbons, Angew. Chem. Int. Ed. 46 (2007) 714-717.

28. Z. F. Li, F. Qin, T. F. Liu, R. Ge, W. Meng, J. H. Tong, S. X. Xiong, Y. H. Zhou, Optical properties and conductivity of PEDOT:PSS films treated by polyethylenimine solution for organic solar cells, Org. Electr. 21 (2015) 144-148.

29. S. Savagatrup, E. Chan, S. M. Renteria-García, A. D. Printz, A. V. Zaretski, T. F. O’Connor, D. Rodriguez, E. Valle, D. J. Lipomi, Plasticization of PEDOT:PSS by common additives for mechanically robust organic solar cells and wearable sensors, Adv. Funct. Mater. 25 (2015) 427-436.

30. Y. Chen, J. H. Xu, Y. J. Yang, Y. T. Zhao, W. Y. Yang, X. L. Mao, X. He, S. B. $\mathrm{Li}$, The preparation and electrochemical properties of PEDOT:PSS/MnO2/PEDOT ternary film and its application in flexible micro-supercapacitor, Electrochim. Acta 193 (2016) 199-205.

31. J. F. Sun, Y. Huang, C. X. Fu, Y. Huang, M. S. Zhu, X. M. Tao, C. Y. Zhi, H. $\mathrm{Hu}$, A high performance fiber-shaped PEDOT@MnO2//C@Fe3O4 asymmetric supercapacitor for wearable electronics, J. Mater. Chem. A 4 (2016) 14877-14883. 
32. Y. Q. Liu, B. Weng, J. M. Razal, Q. Xu, C. Zhao, Y. Y. Hou, S. Seyedin, R. Jalili, G. G. Wallace, J. Chen, High-performance flexible all-solid-state supercapacitor from large free-standing graphene-PEDOT/PSS films, Sci. Rep. 5 (2015) 17045.

33. S. Lehtimaki, M. Suominen, P. Damlin, S. Tuukkanen, C. Kvarnstrom, D. Lupo, Preparation of supercapacitors on flexible substrates with electrodeposited PEDOT/graphene composites, ACS Appl. Mater. Interfaces 7 (2015) 22137-22147.

34. D. Aradilla, D. Azambuja, F. Estrany, M. T. Casas, C. A. Ferreira, C. Alemán, Hybrid polythiophene-clay exfoliated nanocomposites for ultracapacitor devices, J. Mater. Chem. 22 (2012) 13110-13122.

35. D. Aradilla, F. Estrany, D. S. Azambuja, M. T. Casas, J. Puiggalí, C. A. Ferreira, C. Alemán, Conducting poly(3,4-ethylenedioxythiophene)-montmorillonite exfoliated nanocomposites, Eur. Polym. J. 46 (2010) 977-983.

36. M. Culebras, C. Cho, M. Krecker, R. Smith, Y. X. Song, C. M. Gomez, A. Cantero, J. C. Grunlan, High thermoelectric power factor organic thin films through combination of nanotube multilayer assembly and electrochemical polymerization, ACS Appl. Mater. Interfaces 9 (2017) 6306-6313.

37. F. E. Jurin, C. C. Buron, N. Martin, C. Filiatre, Preparation of conductive PDDA/(PEDOT:PSS) multilayer thin film: Influence of polyelectrolyte solution composition, J. Colloid Inter. Sci. 431 (2014) 64-70.

38. S. Lee, M. S. Cho, H. Lee, J. D. Nam, Y. Lee, A facile synthetic route for well defined multilayer films of graphene and PEDOTvia an electrochemical method, J. Mater. Chem. 22 (2012) 1899-1903.

39. S. Kim, S. Y. Kim, J. Kim, J. H. Kim, Highly reliable AgNW/PEDOT:PSS hybrid films: efficient methods for enhancing transparency and lowering resistance and haziness , J. Mater. Chem. C 2 (2014) 5636-5643. 
40. H. Ling, J. L. Lu, S. Phua, H. Liu, L. Liu, Y. Z Huang, D. Madler, P. S. Lee, X. H. Lu, One-pot sequential electrochemical deposition of multilayer poly $(3,4-$ ethylenedioxythiophene):poly(4-styrenesulfonic acid)/tungsten trioxide hybrid films and their enhanced electrochromic properties, J. Mater. Chem. A 2 (2014) 2708-2717.

41. H. J. Lee, G. Anoop, H. J. Lee, C. Kim, J. W. Park, J. Choi, H. Kim, Y. J. Kim, E. Lee, S. G. Lee, Y. M. Kim, J. H. Lee, J. Y. Jo, Enhanced thermoelectric performance of PEDOT:PSS/PANI-CSA polymer multilayer structures, Ener. Env. Sci. 9 (2016) 2806-2811.

42. F. Estrany, D. Aradilla, R. Oliver, E. Armelin, C. Aleman, Properties of nanometric and submicrometric multilayered films of poly(3,4-ethylenedioxythiophene) and poly( N-methylpyrrole), Eur. Polym. J. 44 (2008) 1323-1330.

43. R. Oliver, A. Munoz, C. Ocampo, C. Aleman, F. Estrany, Electrochemical characteristics of copolymers electrochemically synthesized from $\mathrm{N}$-methylpyrrole and 3,4-ethylenedioxythiophene on steel electrodes: Comparison with homopolymers, Chem. Phys. 328 (2006) 299-306.

44. D. Amoura, M. Sánchez-Jiménez, F. Estrany, L. Makhloufi, C. Alemán, Clay incorporation at the dielectric layer of multilayer polymer films for electrochemical activation, Polym. Eur. J. 69 (2015) 296-307.

45. J. Poater, J. Casanovas, M. Solà, C. Alemán, Examining the planarity of poly(3,4-ethylenedioxythiophene): Consideration of self-rigidification, electronic, and geometric effects, J. Phys. Chem. A 114 (2010) 1023-1028.

46. C. Ocampo, C. Alemán, R. Oliver, M. L. Arnedillo and O. Ruíz, Copolymers of $N$-methylpyrrole and 3,4-ethylenedioxythiophene: structural, physical and electronic properties, Polym. Int. 56 (2007) 803-809. 
Table 1. Thickness of the films studied in this work as a function of the chemical composition and the polymerization time..

\begin{tabular}{|c|c|c|}
\hline Film & Polymerization time & Thickness $(\mu)$ \\
\hline \multirow[t]{4}{*}{ PEDOT } & $\theta=100 \mathrm{~s}$ & 1.40 \\
\hline & $\theta=300 \mathrm{~s}$ & 3.94 \\
\hline & $\theta=400 \mathrm{~s}$ & 4.92 \\
\hline & $\theta=450 \mathrm{~s}$ & 5.31 \\
\hline \multirow[t]{3}{*}{ PNMPy } & $\theta=100 \mathrm{~s}$ & 1.55 \\
\hline & $\theta=300 \mathrm{~s}$ & 4.49 \\
\hline & $\theta=450 \mathrm{~s}$ & 6.02 \\
\hline Poly(EDOT-co-NMPy) & $\theta=100 \mathrm{~s}$ & 1.53 \\
\hline \multirow[t]{6}{*}{ 31-PEDOT/PNMPy } & $\theta_{1,3}=100 \mathrm{~s}, \theta_{2}=100 \mathrm{~s}$ & 4.34 \\
\hline & $\theta_{1,3}=200 \mathrm{~s}, \theta_{2}=100 \mathrm{~s}$ & 6.95 \\
\hline & $\theta_{1,3}=100 \mathrm{~s}, \theta_{2}=300 \mathrm{~s}$ & 7.28 \\
\hline & $\theta_{1,3}=100 \mathrm{~s}, \theta_{2}=450 \mathrm{~s}$ & 8.81 \\
\hline & $\theta_{1,3}=300 \mathrm{~s}, \theta_{2}=100 \mathrm{~s}$ & 9.42 \\
\hline & $\theta_{1,3}=450 \mathrm{~s}, \theta_{2}=100 \mathrm{~s}$ & 12.03 \\
\hline 31-PEDOT/P(EDOT-co-NMPy) & $\theta_{1,3}=200 \mathrm{~s}, \theta_{2}=100 \mathrm{~s}$ & 6.93 \\
\hline
\end{tabular}




\section{CAPTIONS TO FIGURES}

Figure 1. (a) Cyclic voltammograms after two and fifty (solid and dashed lines, respectively) consecutive redox cycles and (b) variation of the accumulated voltammetric charge $\left(Q\right.$, in $\left.\mathrm{C} / \mathrm{cm}^{2}\right)$ against the number of consecutive oxidationreduction cycles for: PEDOT (black), PNMPy (red) and poly(EDOT-co-NMPy) (blue). Voltammograms were recorded in acetonitrile solution with $0.1 \mathrm{M} \mathrm{LiClO}_{4}$ using a scan rate of $50 \mathrm{mV} \cdot \mathrm{s}^{-1}$. Initial and final potentials: $-0.50 \mathrm{~V}$; Reversal potential: $1.60 \mathrm{~V}$.

Figure 2. Representative height AFM image $\left(5 \times 5 \mu \mathrm{m}^{2}\right)$ and high resolution SEM micrograph of (a) PEDOT, (b) PNMPy and (c) poly(EDOT-co-NMPy) obtained using $\theta=100 \mathrm{~s}$. The AFM phase image is also displayed for the copolymer.

Figure 3. (a) Cyclic voltammograms after two and fifty (solid and dashed lines, respectively) consecutive redox cycles and (b) variation of the accumulated voltammetric charge $\left(Q\right.$, in $\left.\mathrm{C} / \mathrm{cm}^{2}\right)$ against the number of consecutive oxidationreduction cycles for: PEDOT (black), 31-PEDOT/PNMPy (red) and 31PEDOT/P(EDOT-co-NMPy) (blue). Voltammograms were recorded in acetonitrile solution with $0.1 \mathrm{M} \mathrm{LiClO}_{4}$ using a scan rate of $50 \mathrm{mV} \cdot \mathrm{s}^{-1}$. Initial and final potentials: $0.50 \mathrm{~V}$; Reversal potential: $1.60 \mathrm{~V}$. (c) Chronopotentiometry at $1.0 \mathrm{~mA}$ during $500 \mathrm{~s}$ for PEDOT, 31-PEDOT/PNMPy and 31-PEDOT/P(EDOT-co-NMPy.

Figure 4. Scheme illustrating the behavior of (a) 31-PEDOT/PNMPy and (b) 31$\mathrm{PEDOT} / \mathrm{P}($ EDOT-co-NMPy) supercapacitors in which the intermediate layer acts as a conventional dielectric layer and a thin reservoir filled of heterogeneously distributed nanometric supercapacitors, respectively. The AFM phase image $\left(1.5 \times 5 \mu \mathrm{m}^{2}\right)$ corresponds to the poly(EDOT-co-NMPy) intermediate layer.

Figure 5. Characterization through charging and discharging actions of 31PEDOT/PNMPy films produced using: (a-c) different $\theta_{2}$ values while $\theta_{1,3}$ was 
maintained at $100 \mathrm{~s}$; and (d-f) different $\theta_{1,3}$ values while $\theta_{2}$ was maintained at $100 \mathrm{~s}$. (a,d) Temporal evolution of the accumulated voltage across the films during the charging process. (b,e) Temporal evolution of the current across the films during the charging process. $(\mathrm{c}, \mathrm{f})$ Charge released during the discharging process. A power supply of $6 \mathrm{~V}$ was used for the charge process.

Figure 6. Characterization through charging and discharging actions of 31PEDOT/PNMPy and 31-PEDOT/P(EDOT-co-NMPy) films produced using while $\theta_{1,3}=$ $450 \mathrm{~s}$ and $\theta_{2}=100 \mathrm{~s}$ : (a) Temporal evolution of the accumulated voltage across the films during the charging process; (b) Temporal evolution of the current across the films during the charging process; (c) Charge released during the discharging process; and (d) Charge-discharge curves used to obtain the Coulombic efficiency (see text). A power supply of $6 \mathrm{~V}$ was used for the charge process.

Figure 7. (a) Photograph of the 31-PEDOT/P(EDOT-co-NMPy) OESC used to power the red LED bulb. The photograph shows the LED's ignition circuit, with the two galvanic cells based on the 31-PEDOT/P(EDOT-co-NMPy) prepared electrodes, joined together in series by one of the black cables with crocodile clips at the ends, each pressing one of the two electrodes of each cell. In each cell, the other electrode is pressed with another clip, the one ending the second black cable (cell on the left side of the image) or the one ending the red cable (cell on the right side of the image). The red cable is tethered to the green cable, which can be seen at the bottom right of the image and can be connected to the second red cable that together with the second black cable, indicated above, close the circuit with the LED. The two galvanic cells in series are charged through the connections of the red and black cables that are seen in the back of the image, that associate one of the two electrodes of each cell with the power supply. Once the device has been charged, one of the two indicated cables is disconnected from 
the power supply and the green cable is connected to the red cable of the LED. The timing starts at the same instant that the discharge circuit of the galvanic cells with the LED is closed. (b) Variation of the voltage during the charging-discharging processes of the OESC. For the discharge a resistance of $1 \Omega$ was used. (c) Charge accumulated and released during the charging and discharging processes, respectively. (d) Red LED bulb powered. A power supply of $12.3 \mathrm{~V}$ was used for the charge process. 

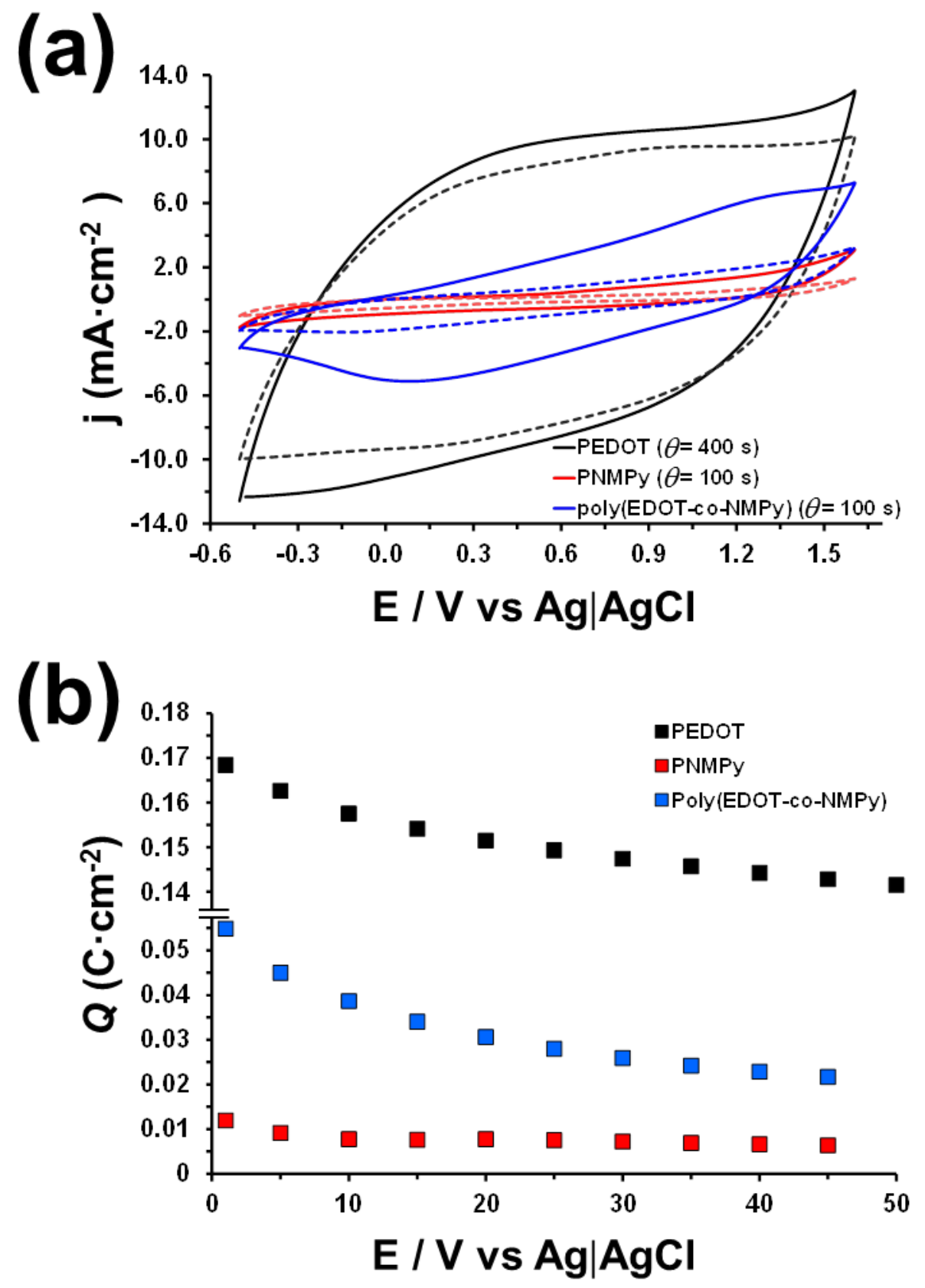

Figure 1 

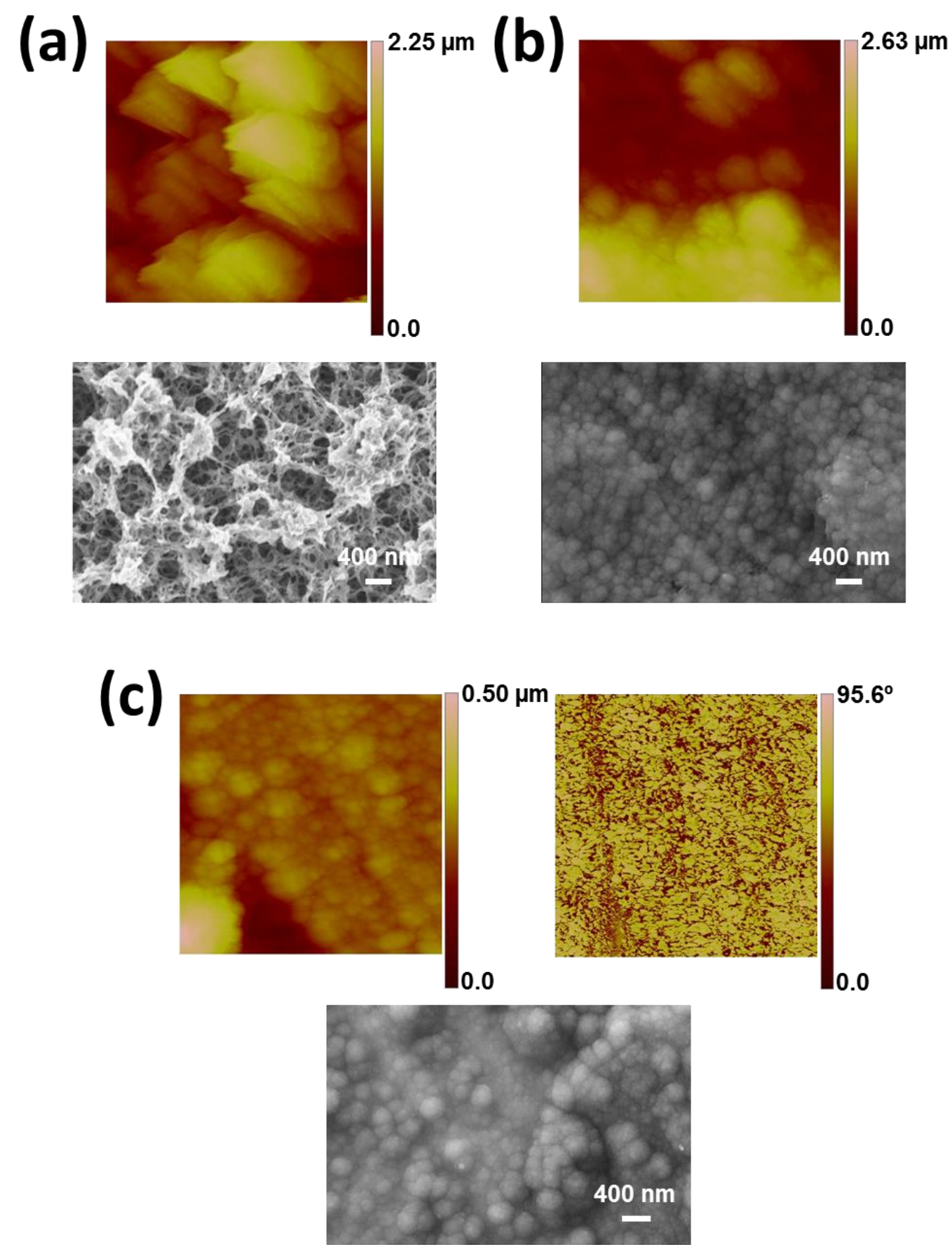

Figure 2 
(a)

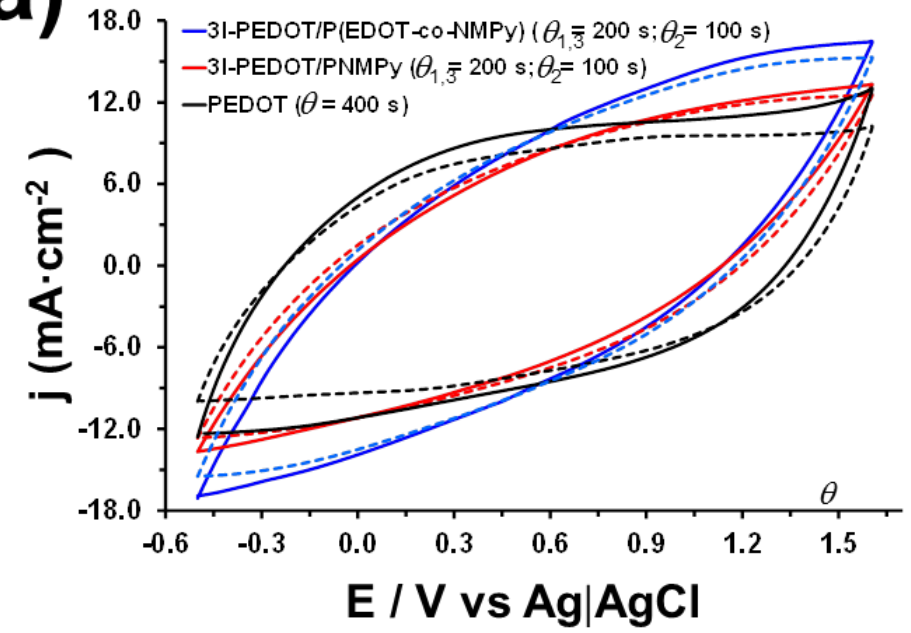

(b)

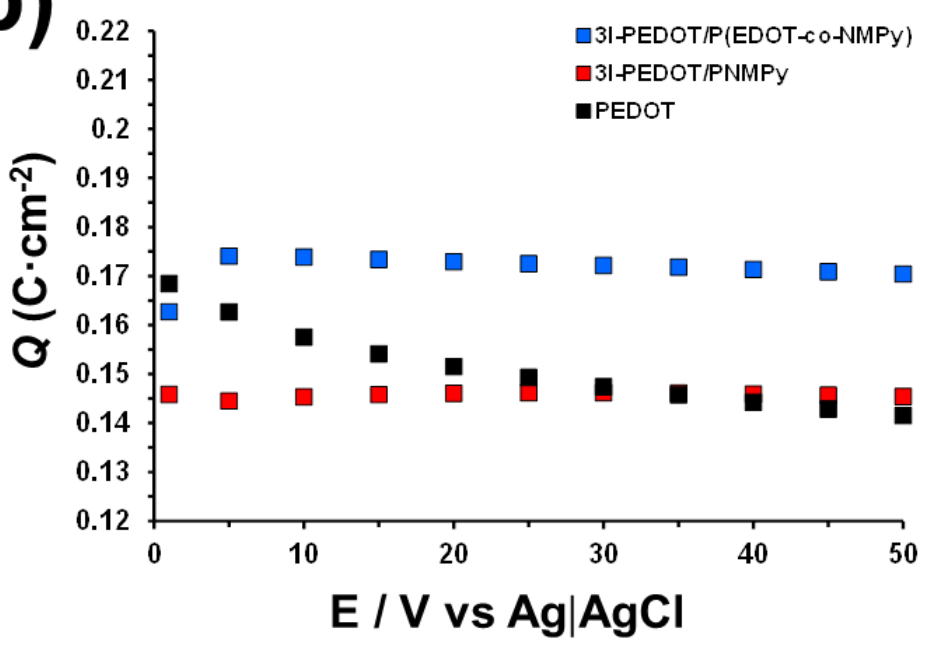

(c)

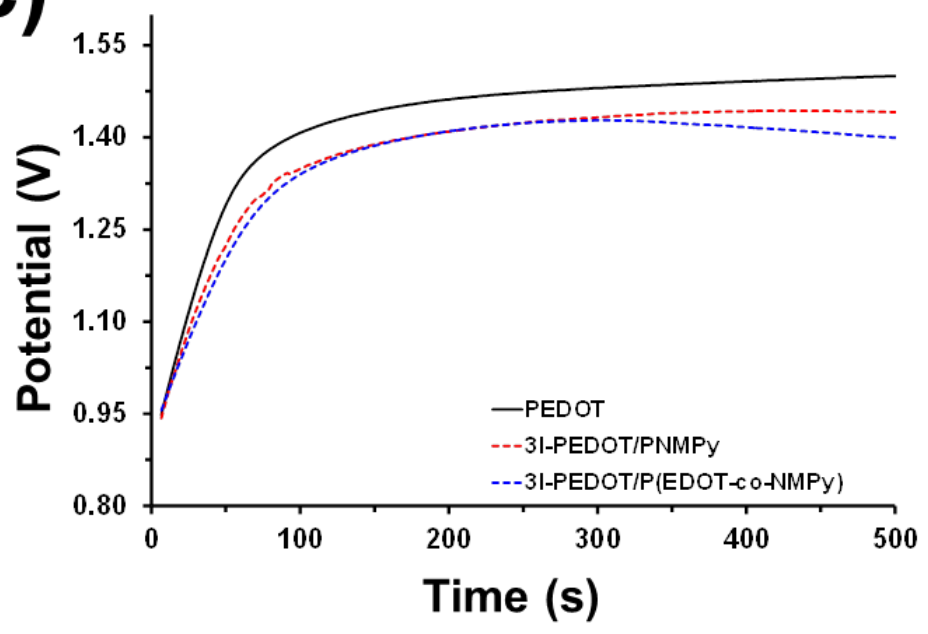

Figure 3 


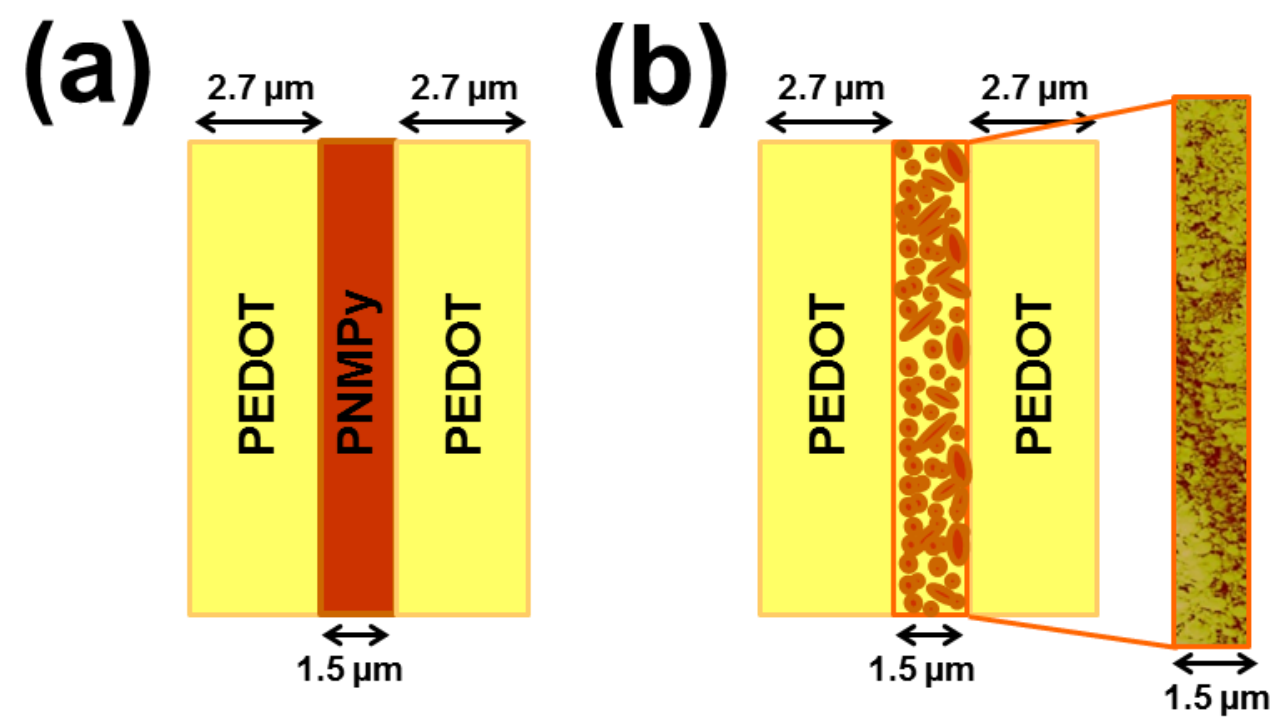

Figure 4 
(a)

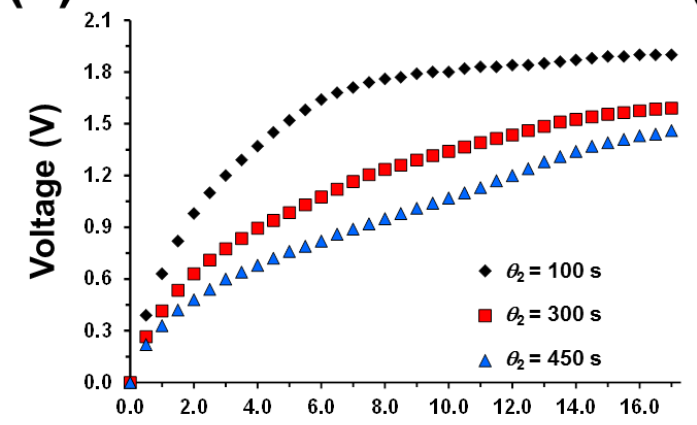

(b)

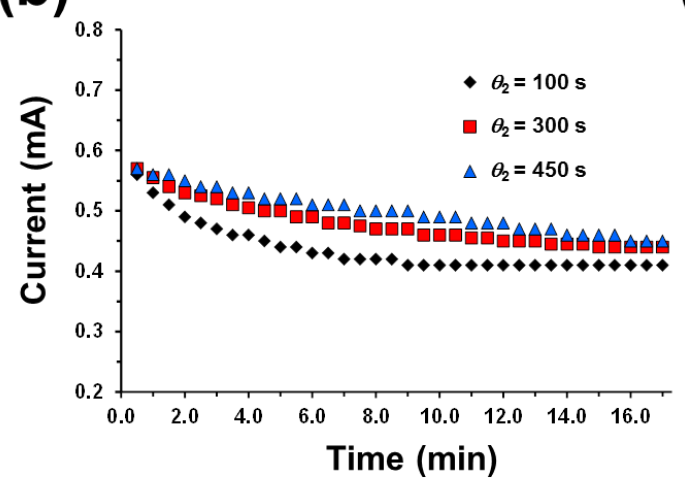

(c)

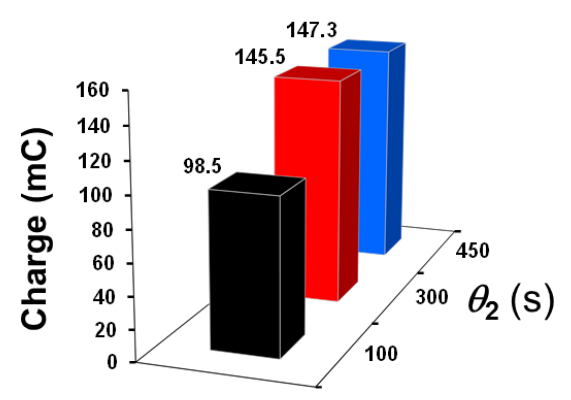

(d)

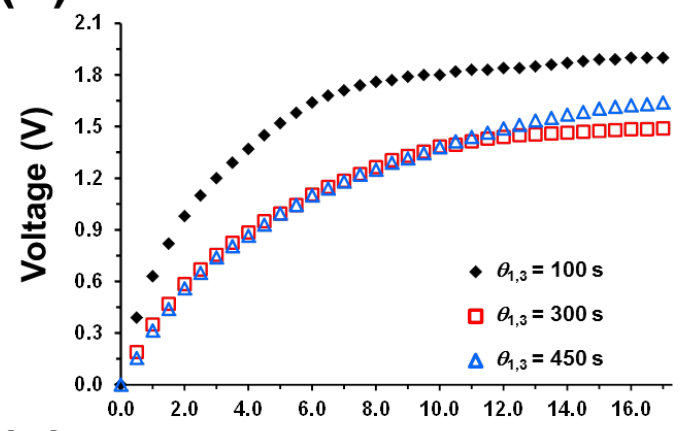

(e)

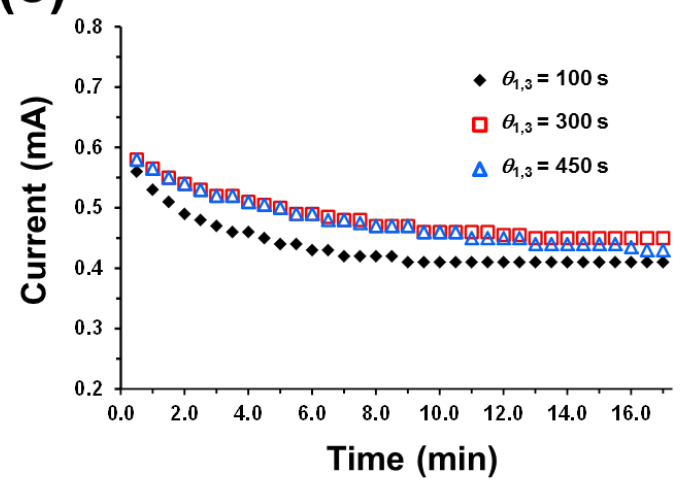

(c)

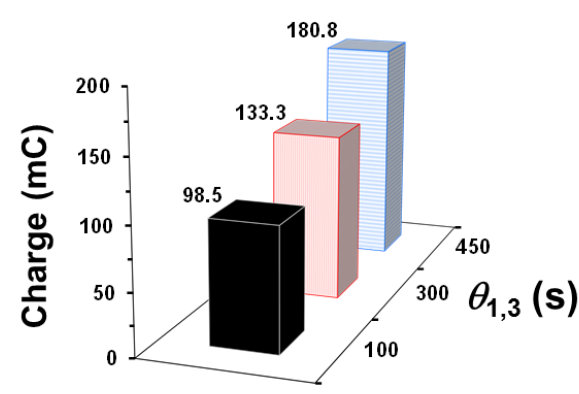

Figure 5 
(a)

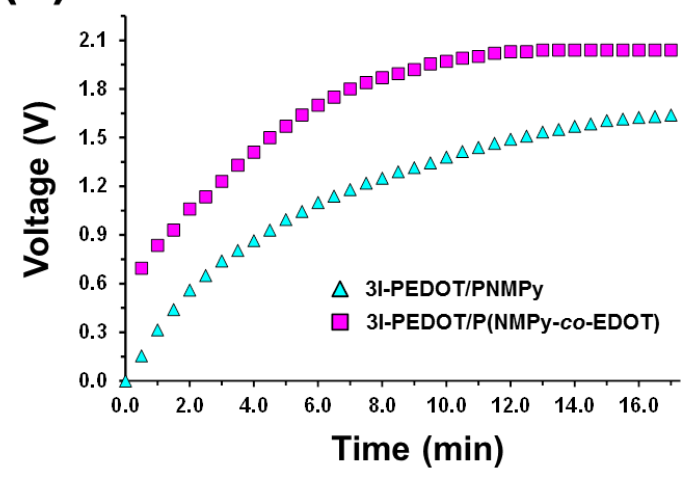

(c)

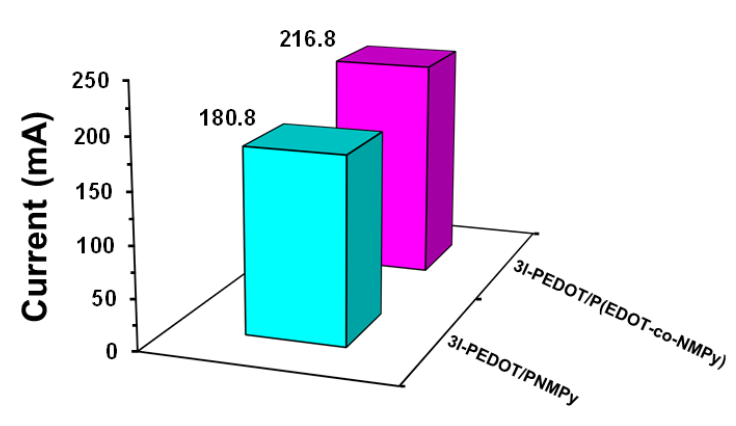

(b)

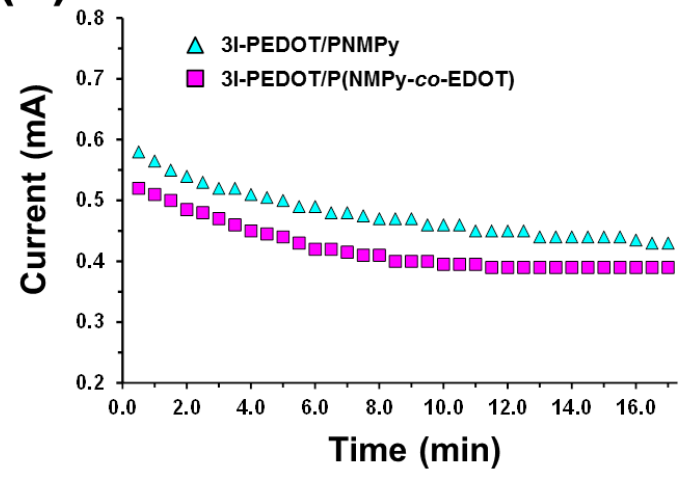

(d)

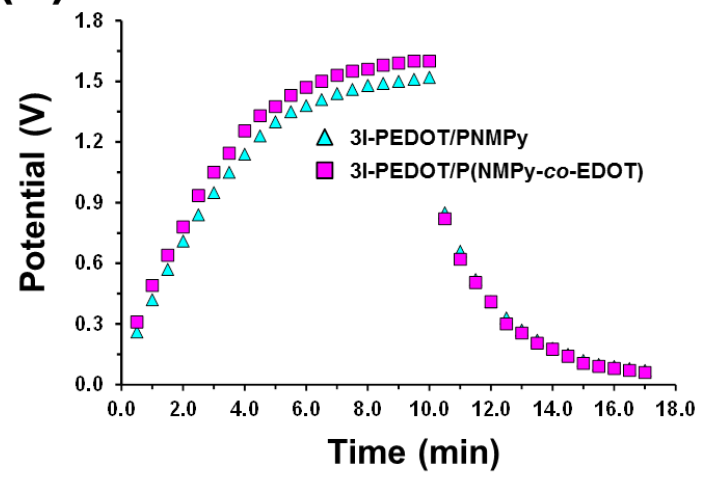

Figure 6 
(a)

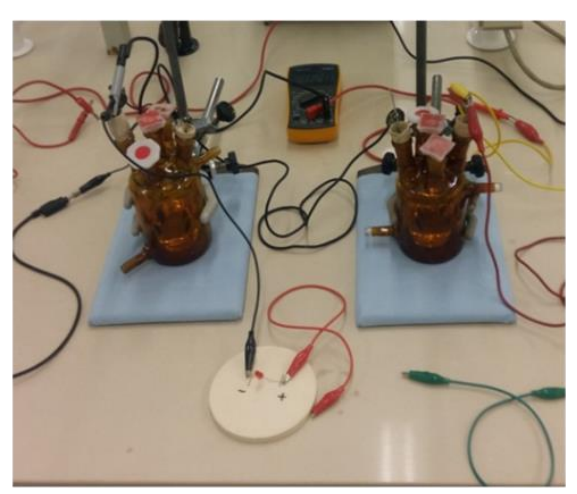

(b)

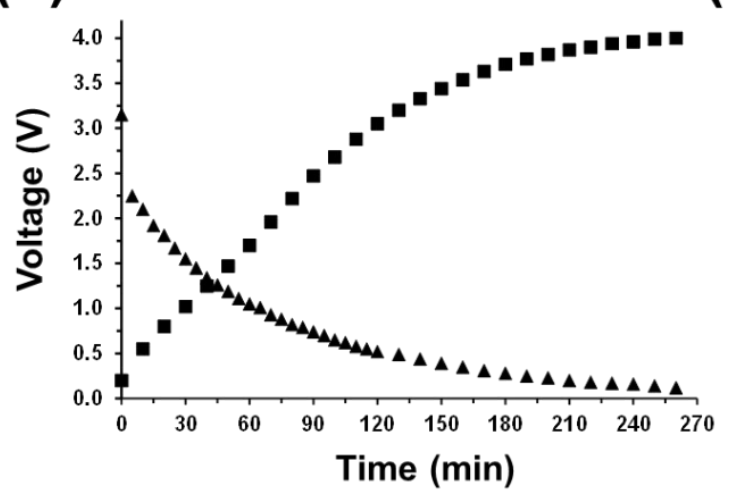

(c)

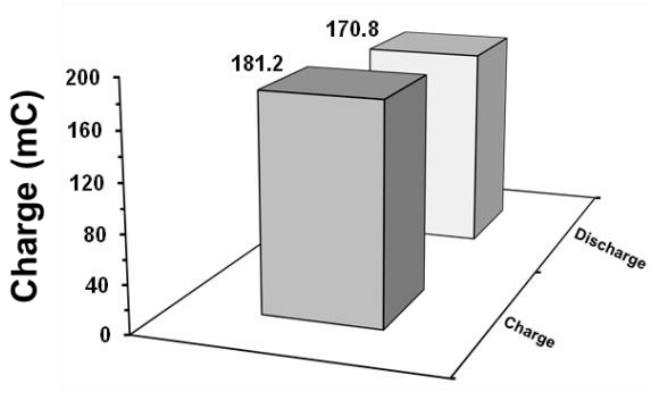

(d)

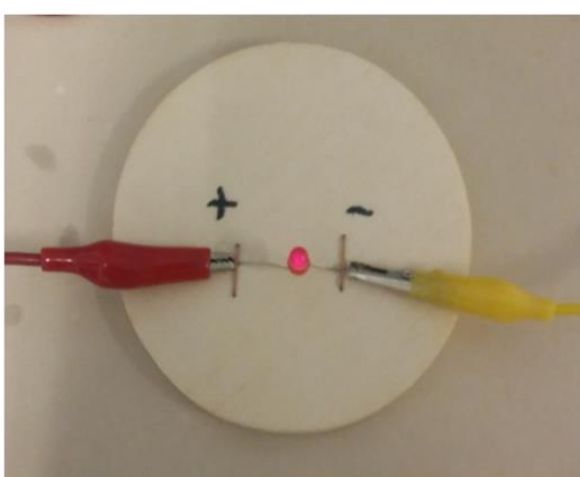

Figure 7 


\section{Graphical Abstract}

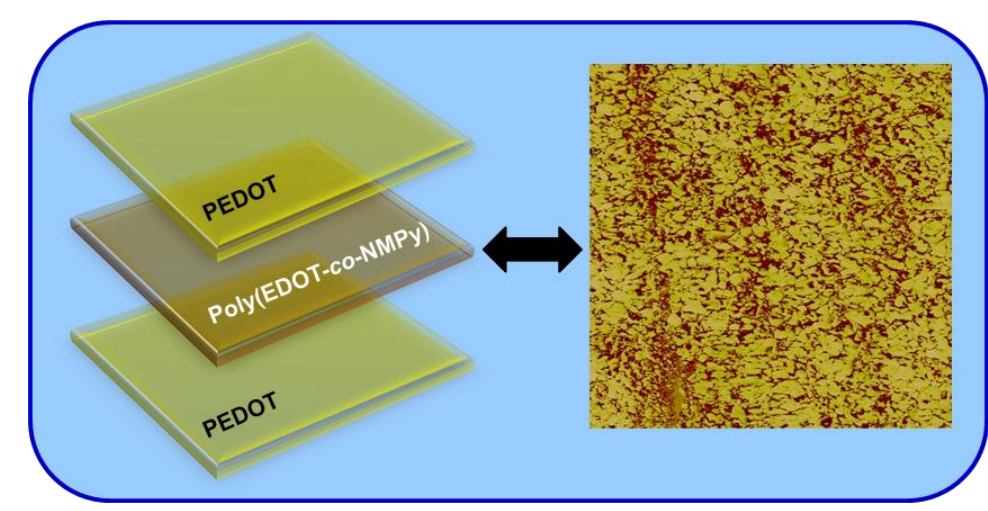

